\title{
The Design of Greenhouse Environment Control System Based on Variable Universe Fuzzy Control Algorithm
}

\author{
Haiyan He and Heru Xue* \\ College of computer \& information Engineering, \\ Inner Mongolia Agricultural University, \\ Hohhot, Inner Mongolia, P.R. China \\ hehaiyan808@sina.com, xuehr@imau.edu.cn
}

\begin{abstract}
Greenhouse environment has the characteristics such as multipleinput, multiple-output, nonlinearity, and difficulties of establishing its accurate mathematical model. Therefore, in order to make the greenhouse environment control more effective and accurate, an expert system of variable universe fuzzy control based on BP neural network has been proposed. In this method an appropriate universe is selected according to the error and the changing rate of error to overcome the effect for greenhouse control when the error is too large or too small. The paper introduces the design of greenhouse environment control system based on variable universe fuzzy control in details, and presents the main control parameters.
\end{abstract}

Keywords: fuzzy control, variable universe, greenhouse environment control, expert system, BP neural network.

\section{Introduction}

Greenhouse environment control can increase crop yield, improve quality, adjust the production cycle and improve the economic efficiency, if we can make full use of natural resources and change environmental factors (e.g. the temperature, humidity, light intensity, $\mathrm{CO}_{2}$ concentration, etc.) to obtain the optimum condition of crop growth[1]. The current greenhouse environment controlling system has a lot of shortcomings, especially great influence on the control accuracy of the controlling algorithm. Only reasonable control algorithm can make the comprehensive factor of greenhouse environment achieve optimal control effect, and make the greenhouse system achieve intelligent level.

There are a lot of algorithms about greenhouse environment control, such as PID control algorithm, the fuzzy control algorithm, the neural network control algorithm, etc. However, these algorithms have their shortcomings. PID control algorithm needs to build accurate mathematical model and it is difficult to build accurate mathematical

${ }^{*}$ Corresponding author. 
model for the multivariable and complex greenhouse environment. With inaccurate model, the entire greenhouse environment control system will be directly affected, even some errors occur in the system. Fuzzy control doesn't need accurate mathematical model. Computer is used to achieve the control which people complete with natural language. But there are very high requirements on membership functions selection and expert knowledge. If not taken carefully, it will be inaccurate and lead to the influence of greenhouse crop growth.

In this paper, the variable universe fuzzy control based on BP neural network is used in greenhouse environment control system, which has strong dynamic prediction functions and decision-making explained ability.

\section{Variable Universe Fuzzy Control Based on BP Neural Network}

\subsection{Fuzzy Control}

Fuzzy control doesn't need accurate mathematical model. Computer is used to achieve the control which people complete with natural language. The control algorithm puts all environmental parameters together, then realizes fuzzy control. The block diagram of the principle of fuzzy control system is shown as fig.1 [2].

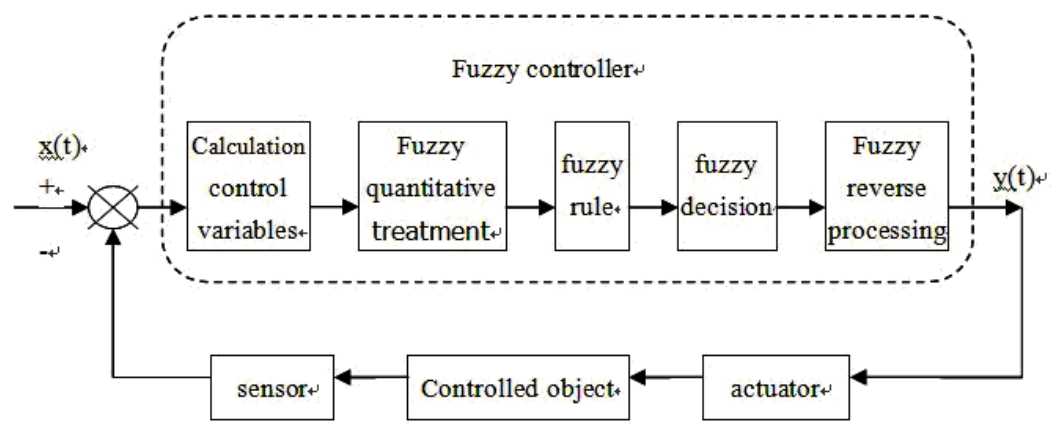

Fig. 1. The block diagram of principle of fuzzy control system

\subsection{Variable Universe Fuzzy Control}

In greenhouse environment control process, greenhouse environment is a multivariable nonlinear system, which is difficult to establish the mathematical model. Using the fuzzy control doesn't need to build accurate mathematical model, but the initial variable universe has great effect on the accuracy of control system. As a result, Variable Universe Fuzzy Control can solve the problem of selecting initial universe.

On the premise of fuzzy division unchanged in the variable universe, Variable Universe shrinks with error decrease, and expands with error increase. Through the changing of universe, it makes the initial rule which experts concluded a more 
effective new rule. On the surface, the number of rules has not changed, but the rules are more precise because the universe changes, equivalent to increase of the number of rules, and improvement of the accuracy of control [3]. The change of domain is shown as fig. 2 .
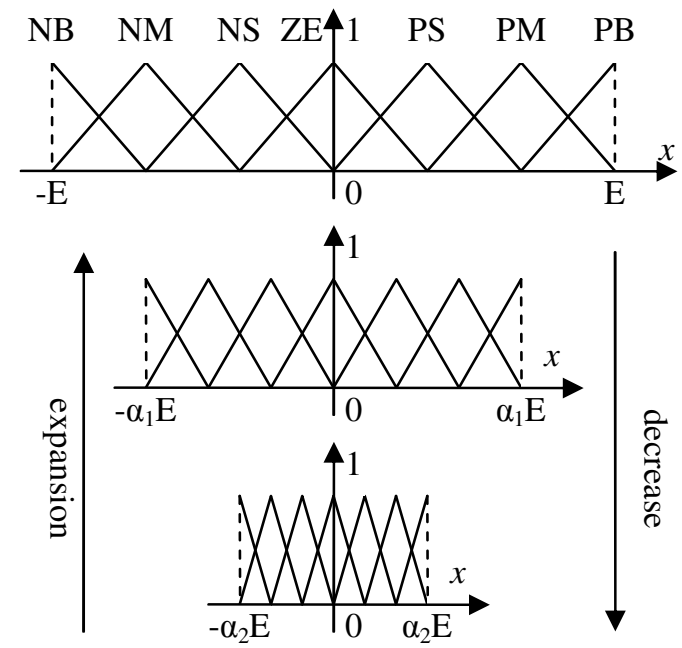

Fig. 2. Change of universe chart

\subsection{BP Neural Network}

The BP neural network is Error Back Propagation network. It consists of three parts: input layer, hidden layer and output layer. The BP neural network, which realizes adjustable factor function of universe, is a three-layer forward network composed of a binary input and a triple output. The network's input node is error $e$ and error rate $e c$, and the output node is adjustable factors of systematology universe, $\alpha 1, \alpha 2, \beta$. The structure of BP neural network is shown as fig.3 [4].

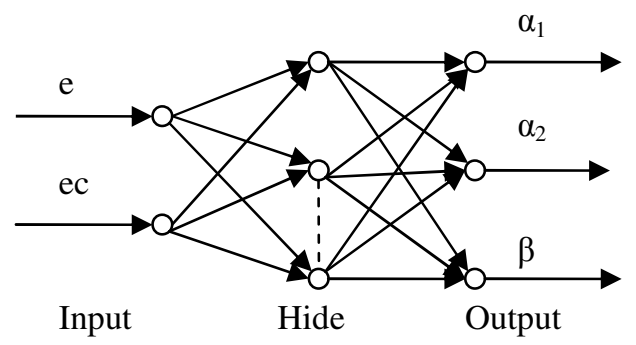

Fig. 3. BP neural network structure chart

\subsection{Variable Universe Fuzzy Control Based on BP Neural Network}

Variable universe fuzzy control based on BP neural network is composed of fuzzy control by fuzzy control technology and the BP neural network. It combines fuzzy 
reasoning ability of linguistic expression with the BP neural network self-learning ability. The BP neural network describes the telescopic change. In system control process, the BP neural network computes out suitable factor. Fuzzy control change universe achieves better control effect according to the suitable factor. The structure of variable universe fuzzy control based on BP neural network is shown as fig.4 [5-6].

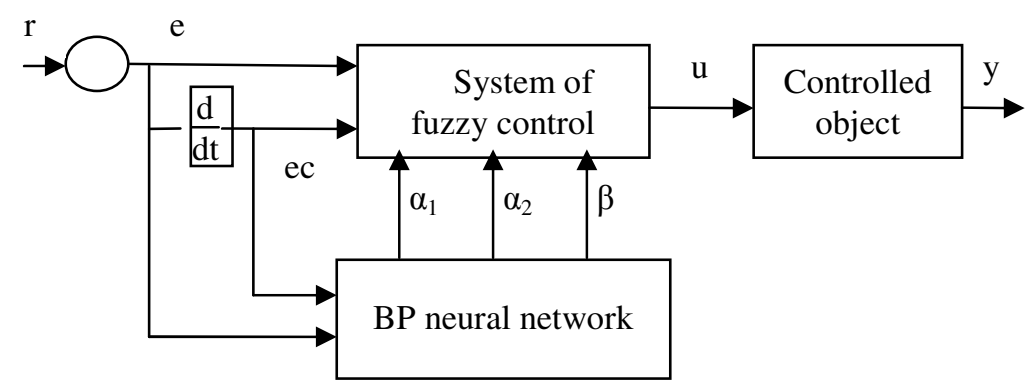

Fig. 4. Structure chart of variable universe fuzzy control based on BP Neural Network

\section{Controlling System Design}

\subsection{Structure of Control System}

Indoor/outdoor sensor array collects real-time environmental data, such as temperature, humidity and so on. The data are analyzed by variable universe fuzzy control based on BP neural network system, and control decision is obtained, then the control part is notified to perform corresponding action. Control system structure is shown as fig.5 [7].

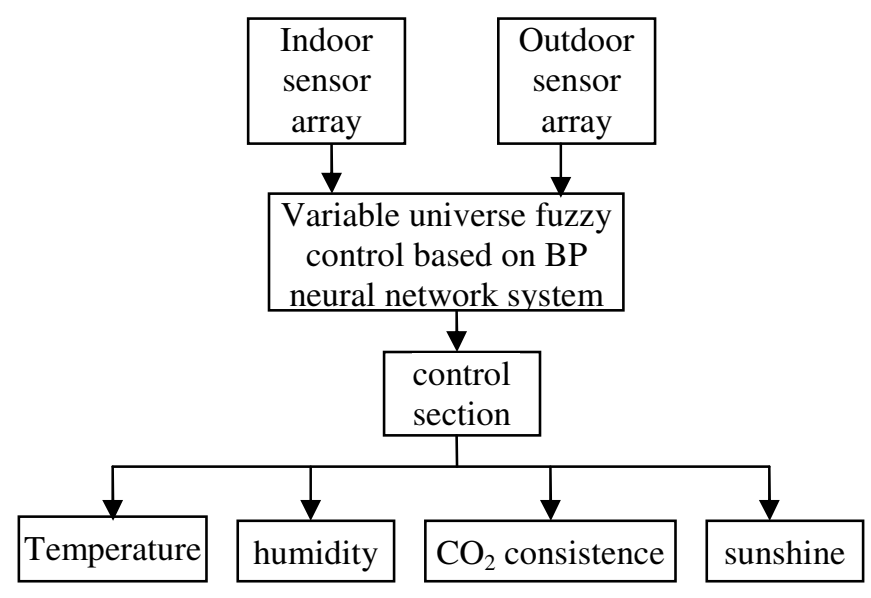

Fig. 5. Control system structure chart 


\subsection{Control System Process}

Greenhouse system is a big lag and nonlinear systems, so we need to constantly detect greenhouse system control parameters, and adjust the execution time of each execute equipment, which makes greenhouse always maintain the best environment for crop growth. Control system process is shown as fig.6 [8].

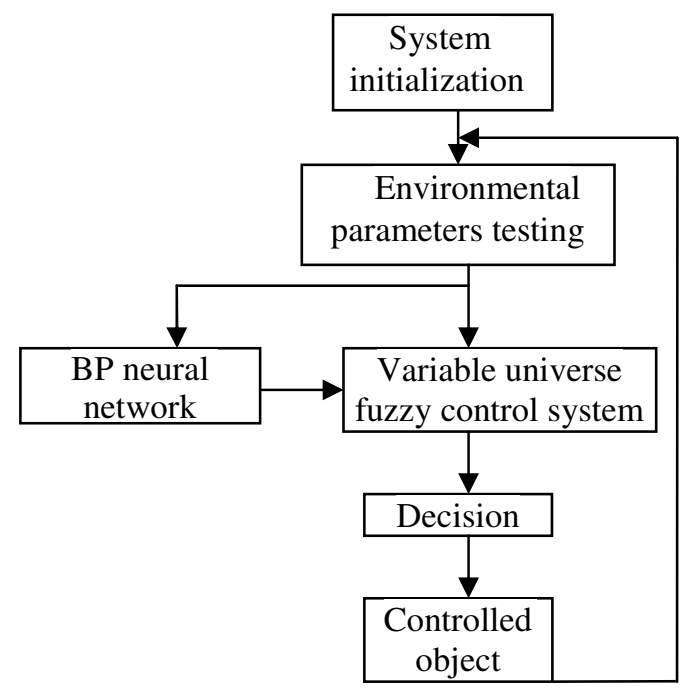

Fig. 6. Control system process chart

\subsection{Control System Parameter Design}

\subsubsection{Variable Universe Fuzzy Control Design}

Error $e$ basic universe $[-4,+4]$;

Error rate $e c$ basic universe $[-2,+2]$;

Output $\mathrm{U}$ basic universe $[-1,1]$; Controlled variable of output is described by four fuzzy states, for instance PB, PM, PS, ZO.

The principle of selecting controlled variables: When the error is big, we should primarily choose controlled variable to eliminate error as soon as possible; when the

Table 1. Fuzzy control rule

\begin{tabular}{llllllll}
\hline \multirow{2}{*}{ E } & \multicolumn{7}{c}{ EC } \\
\cline { 2 - 7 } & NB & NM & NS & ZO & PS & PM & PB \\
\hline NB & PB & PB & PB & PM & PS & ZO & ZO \\
NM & PB & PB & PM & PM & PS & ZO & ZO \\
NS & PB & PB & PM & PS & ZO & NS & NM \\
ZO & PM & PM & PS & ZO & NS & NM & NM \\
PS & PM & PS & ZO & NS & NM & NM & NM \\
PM & PM & ZO & ZO & NM & NM & NM & NM \\
PZ & PS & ZO & ZO & NB & NB & NB & NB \\
\hline
\end{tabular}


error is less, we should avoid overshooting while selecting controlled variables, and ensure the stability of the system.

We choose triangle membership Functions, due to variable universe fuzzy control having low requirement to membership function, which is denoted by three parameters $(\mathrm{a}, \mathrm{b}, \mathrm{c})$ :

$$
u(x)= \begin{cases}0 & x<=a \\ \frac{x-a}{b-a} & a<x<=b \\ \frac{x-b}{c-b} & b<x<=c \\ 1 & x>=c\end{cases}
$$

\subsubsection{BP Neural Network Design}

There are 2 input nodes, 3 output nodes, 7 hidden layer nodes. Learning rate $\eta$ is 0.4 , momentum factor is 0.1 and mean square error is 0.01 .

\section{Output Results Comparison}

Variable universe fuzzy control which is based on BP neural network, according to the size of the error and error rate, chooses suitable universe to control. It solves the improper selection problem of initial universe to obtain more accurate control. The following is a comparison of output between fuzzy control and variable universe fuzzy control based on BP neural network (e.g. temperature).

Table 2. Output comparison

\begin{tabular}{|c|c|c|c|c|c|}
\hline \multirow{2}{*}{ Error } & \multirow{2}{*}{$\begin{array}{l}\text { Error } \\
\text { rate }\end{array}$} & \multicolumn{2}{|c|}{$\begin{array}{l}\text { variable universe fuzzy } \\
\text { control }\end{array}$} & \multicolumn{2}{|c|}{ fuzzy control } \\
\hline & & $\begin{array}{c}\text { Output } \\
\mathrm{U}\end{array}$ & $\begin{array}{c}\text { Input } \\
\text { energy }(\mathrm{W})\end{array}$ & $\begin{array}{l}\text { Output } \\
\mathrm{U}\end{array}$ & $\begin{array}{c}\text { Input } \\
\text { energy }(W)\end{array}$ \\
\hline-3.5 & -1 & 5.00 & $4.8 \times 10^{5}$ & 6.00 & $4.8 \times 10^{5}$ \\
\hline-2.2 & -1.2 & 4.28 & $3.2 \times 10^{5}$ & 6.00 & $4.8 \times 10^{5}$ \\
\hline-2.5 & -0.8 & 4.58 & $4.8 \times 10^{5}$ & 5.20 & $4.8 \times 10^{5}$ \\
\hline-2.0 & -0.5 & 4.00 & $3.2 \times 10^{5}$ & 4.00 & $3.2 \times 10^{5}$ \\
\hline-1.8 & -1.2 & 3.50 & $3.2 \times 10^{5}$ & 6.00 & $4.8 \times 10^{5}$ \\
\hline-1.5 & -0.8 & 3.00 & $1.6 \times 10^{5}$ & 5.20 & $4.8 \times 10^{5}$ \\
\hline-1.2 & -0.7 & 2.70 & $1.6 \times 10^{5}$ & 4.80 & $4.8 \times 10^{5}$ \\
\hline-0.8 & -0.3 & 1.82 & $1.6 \times 10^{5}$ & 2.66 & $1.6 \times 10^{5}$ \\
\hline-0.3 & -0.1 & 0.70 & 0 & 1.16 & $1.6 \times 10^{5}$ \\
\hline
\end{tabular}


It is very important for the rational choice of suitable factor for input and output variables of the fuzzy control. Suitable factors $\alpha 1$ and $\alpha 2$ are the different weight of error and the error rate of input variable. So it has great influence on the dynamic performance of the control system. Variable universe fuzzy control based on BP neural network has solved this problem. It gives suitable factor which adapts with error and error rate by the BP neural network. It makes the control more accurate, and won't make the responding time too long or overshoot the phenomenon.

As shown in chart 2 , When the error and error rate is $(-2.2,-1.2) 、(-1.5,-0.5) 、(-$ $1.2,-0.7)$, fuzzy control output $(\mathrm{U}=6)$ reaches the highest level $(4.8 \times 105 \mathrm{~W})$. Big energy input may cause overshooting and make the concussion intensified. Therefore, fuzzy control variable universe based on BP neural network is better than fuzzy control. For a multivariate and nonlinear greenhouse environment system with delay phenomenon, variable universe is more suitable.

\section{Conclusion}

As stated previously, variable universe fuzzy control based on BP neural network is more suitable for greenhouse environment control. The system can gain better control effect than general control system. If we ascertain the change rule of real-time of universe. In addition, variable universe fuzzy control based on BP neural network also does not need too much expert knowledge, which makes the establishment of the greenhouse environment control system easier.

Acknowledgements. This study has been funded by Research projects (ZN201010 and NDPYTD2010-9).

\section{References}

1. Li, X., Yang, M., Yang, R.: Modern greenhouse environment intelligent control development present situation and prospect. Journal of Agricultural Mechanization Research (4), 9-12 (April 2008)

2. Zhao, Y.: Study on the expert system of greenhouse intelligent control. Kunming polytechnic university (March 2007)

3. Li, L.: Study on variable universe fuzzy control algorithm. University of electronic science and technology (May 2008)

4. Zhang, H., Chen, P., Liu, X., Yu, Z.: The expert system based on neural network in the application of greenhouse control. Journal of Chengdu University Of Information Technology 25(3), 260-263 (2010)

5. Hayashi, Y., Buckley, J.J., Czogala, E.: Fuzzy expert systems versus neural networks. In: Proc. of Int. Joint Conf. on Neural Networks, Baltimore, MD, June 7-11, vol. 2, pp. 720762 (1992)

6. Wang, L.-X.: Adaptive Fuzzy Systems and Control. Prentice-Hall, Englewood-Cliffs (1994)

7. Mamdani, E.H.: Application of Fuzzy Algorithms for Control of a Simple Dynamic Plant. Proc. IEE 121(12), 1585-1588 (1974)

8. Cai, C.: Study on intelligent greenhouse environment control system. Journal of Chongqing Institute of Technology 21(10), 105-107 (2007) 